\title{
Discriminación y violencia homofóbica en el sistema escolar: estrategias de prevención, manejo y combate
}

JUAN CORNEJO ESPEJO

Universidad Católica del Maule, Chile Santiago, Chile

\section{RESUMEN}

La discriminación por orientación sexual se ha convertido en uno de los problemas más preocupantes de los sistemas escolares contemporáneos, ya sea por los daños psicoemocionales que provoca en las víctimas, como por las consecuencias sociales que distorsiona las relaciones interpersonales y transforma la escuela en un espacio inseguro y poco gratificante para el aprendizaje y el desarrollo personal. A partir de los registros de denuncias de discriminación por orientación sexual del Ministerio de Educación, artículos de prensa y testimonios de víctimas de violencia homofóbica en contextos escolares chilenos, el artículo propone diez estrategias de prevención, manejo y combate de la homofobia escolar. Estrategias que buscan promover e incentivar en las comunidades educativas la reflexión y el diálogo en torno al tema, de modo de desarrollar programas propios de prevención que respondan a sus propias necesidades, intereses y culturas locales.

PALABRAS CLAVE

estrategias; prevención; homofobia; sistema escolar. 


\title{
DISCRIMINATION AND HOMOPHOBIC VIOLENCE IN THE SCHOOL SYSTEM: PREVENTION, STRATEGIES, MANAGEMENT AND COMBAT
}

\begin{abstract}
The discrimination for sexual orientation has become in one of the most worrying problems in the contemporary school systems, either by the psycho-emotional damage it causes to victims as for the social consequences that distorts relationships and transforms school in unsafe and unrewarding space for learning and personal development. From the records of complaints of discrimination by sexual orientation from the Ministry of Education, newspaper articles and testimonies of victims of homophobic violence in Chilean school settings, the article proposes ten prevention strategies, management and combating school homophobia. Strategies that seek to promote and encourage educational communities in reflection and dialogue on the issue, so as to develop their own prevention programs to meet their own needs, interests and cultures.
\end{abstract}

KEYWORDS

strategies; prevention; homophobia; school system.

\section{DISCRIMINAÇÃO E VIOLÊNCIA HOMOFÓBICA \\ NO SISTEMA ESCOLAR: ESTRATÉGIAS DE PREVENÇÃO, GESTÃO E COMBATE}

\section{RESUMO}

A discriminação por orientação sexual tornou-se um dos problemas mais preocupantes dos sistemas escolares contemporâneos, seja pelos danos psicológicos que provoca nas vítimas, seja pelas consequências sociais que distorce as relações interpessoais e transforma a escola em um espaço inseguro e pouco gratificante para a aprendizagem e o desenvolvimento pessoal. Com base nos registros de denúncias de discriminação por orientação sexual do Ministério da Educação, artigos de imprensa e testemunhas de vítimas de violência homofóbica em contextos escolares chilenos, o artigo propõe dez estratégias para a prevenção, gestão e combate da homofobia escolar. Estratégias que visam promover e incentivar nas comunidades educativas a reflexão e o diálogo sobre a questão, a fim de desenvolver programas específicos de prevenção, para atender às suas necessidades, interesses e culturas locais.

PALAVRAS-CHAVE

estratégias; prevenção; homofobia; sistema escolar. 


\section{INTRODUCCIÓN}

En las siguientes páginas se esbozan algunas ideas sobre las cuales se deberían construir estrategias de manejo y prevención de la discriminación y violencia homofóbica en el sistema escolar, las cuales son fruto del discernimiento teórico y análisis de la situación de discriminación homofóbica presentes en el sistema escolar chileno. Estrategias que están en desarrollo en otros contextos educativos, especialmente de países del primer mundo.

En el caso latinoamericano las iniciativas hasta ahora implementadas son más bien focalizadas y no tienen un alcance nacional; sin contar que están escasamente documentadas como ocurre en general con todas las experiencias de inclusión educativa (Artiles y Kozleski, 2014). Con todo, no se puede desconocer que el problema de la discriminación motivada por la orientación sexual y/o identidades genéricas y su expresión inmediata, el bullying homofóbico, aún es un tema pendiente, que dista mucho de estar resuelto aún en países con mayor trayectoria en el tema.

En Chile las dificultades para abordar temáticas asociadas a la sexualidad no solo se explican por las resistencias de los grupos conservadores, muchos de ellos de cuño religioso, invisibilización o silenciamiento de las disidencias y un doble estándar en materia de sexualidad, sino también por los desacuerdos entre los distintos actores sociales respecto de cuál es la educación sexual que se debería impartir en los establecimientos educacionales del país. En ese contexto no resulta extraño que no exista un plan único de sexualidad, sino que cada establecimiento puede entregar la educación sexual que desee o aún omitirla. En la actualidad, existen siete programas de educación sexual diseñados por distintas universidades u organismos interesados en el tema, que además de no ser obligatorios deben ser comprados por los establecimientos educacionales, situación que además de curiosa introduce claramente un componente mercantil. Es decir, es el establecimiento quien decide que educación sexual impartir, dónde nutrirse de información o con qué proveedor acceder a ella.

Si bien históricamente en la mayoría de los programas de educación sexual o iniciativas formativas el tema de la diversidad sexual ha sido invisibilizado u omitido no se puede eludir el hecho que esa decisión no es azarosa, sino que responde a una orientación que es funcional a la mantención del orden heternormativo. Las escasas veces que el tema surge, normalmente, es para subrayar un supuesto carácter patológico o imponer un discurso moralizante descalificador de las disidencias.

Y pese a que en los últimos años ha habido iniciativas educativas de parte del Movimiento de Liberación e Integración Homosexual (MOVILH), patrocinadas por el Ministerio de Educación (MINEDUC), que buscan sensibilizar acerca del tema de la diversidad, ${ }^{1}$ tales iniciativas además de focalizadas y de alcance muy limitado, no proporcionan en rigor una agenda que permitan construir un programa de educación sexual o delinear estrategias pedagógicas de combate, manejo y

1 Los programas pilotos desarrollados por el MOVILH que buscaban sensibilizar acerca de la diversidad sexual en las escuelas y la homoparentalidad son: Educando en la diversidad: orientación sexual e identidad de género en las aulas (2010); y Nicolás tiene dos papás (2014). 
prevención de la discriminación por orientación sexual y del bullying homofóbico. Lo mismo se puede decir de algunas iniciativas escolares preventivas desarrolladas por la Organización de las Naciones Unidas para la Educación, la Ciencia y la Cultura (UNESCO) en algunas escuelas del Gran Santiago. Tanto el acotado número de escuelas participantes del proyecto, como el escaso impacto de dichas iniciativas a nivel nacional han incidido en el desconocimiento que existe entre los educadores de esas intervenciones.

En lo que respecta a las fuentes de información sobre las cuales se realizó el análisis que sirve de base al artículo destacan: entrevistas en profundidad a estudiantes Lesbianas, Gais, Bisexuales y Transexuales (LGBT), disidentes sexuales, que han sido objeto de discriminación y/o bullying homofóbico; entrevistas a distintos agentes educativos (profesores, directivos, paradocentes, etc.) que han sido testigos o les ha tocado lidiar con este tipo de situaciones en el cumplimiento de sus obligaciones profesionales; registro de denuncias de discriminación homofóbica elaborada por el MINEDUC; y notas de prensa aparecidas en periódicos de circulación nacional (El Mercurio, La Tercera, Las Últimas Noticias y La Cuarta) en la presente década.

Y si bien no se pretende proponer un proyecto único de intervención, dada la diversidad de contextos y problemáticas, que responden a sus propias dinámicas internas y culturas locales, si se pueden esbozar algunas pistas que ayuden a las distintas comunidades escolares a reflexionar, discutir y evaluar sus fortalezas y debilidades en vista de construir sus propios proyectos.

Más que recetas únicas los ejes propuestos ponen su acento en aspectos claves sobre los cuales se deben elaborar las estrategias pedagógicas de prevención, manejo y combate de la homofobia en los espacios escolares.

\section{DISCRIMINACIÓN POR ORIENTACIÓN SEXUAL EN EL SISTEMA ESCOLAR CHILENO}

El análisis de las denuncias de discriminación por orientación sexual registradas por el MINEDUC, en el período comprendido entre 2009-2015, muestra que de las 95 denuncias por este motivo, el 53,8\% se llevó a cabo en las oficinas regionales del MINEDUC en todo el país, en tanto que el 46,1 \% lo hizo a través de la plataforma diseñada para ese propósito. Del total de las denuncias la mayor parte de ellas fue realizada por los apoderados de las víctimas $(38,6 \%)$, seguida por los padres/madres (21,5\%), y en tercer lugar por los propios estudiantes $(15,3 \%)$.

De ese total el $61,5 \%$ eran mujeres y solo un $38,4 \%$ eran hombres, lo cual demuestra que las mujeres disidentes sexuales y sus familias parecieran estar más dispuestas a denunciar, a diferencia de los varones cuyas familias justifican sus denuncias diciendo que el comportamiento o actitudes de sus hijos fueron mal interpretados por parte de sus profesores y compañeros. Es decir, del conjunto de las denuncias se percibe que las estudiantes asumen abiertamente una orientación sexual distinta a la heterosexual, a diferencia de sus compañeros que no solo no asumen una orientación disidente, sino que insisten que fueron confundidos con homosexuales. 
Lo anterior deja en evidencia que los estudiantes y sus familias no pretenden evidenciar la discriminación sexual presente en las escuelas chilenas y consecuentemente no cuestionan el orden heteronormativo imperante (Butler, 2002), a diferencia de las estudiantes que reivindican una identidad lesbiana y su derecho al reconocimiento social al interior de la escuela.

Otro antecedente de interés que puede ayudar a explicar lo generalizado del problema de la discriminación por orientación sexual es que las denuncias se distribuyen a lo largo de todo el país. No obstante, el mayor número de ellas se concentra en la Región Metropolitana de Santiago (53,3\%) y en Valparaíso (21,5\%). Concentración explicable porque son esas ciudades las más urbanizadas y las que concentran los núcleos poblacionales más importantes del país.

Asimismo, si bien es posible hallar denuncias en casi todos los niveles de la educación básica y en la totalidad de enseñanza media, el grueso de ellas se concentra en los dos últimos años de la educación básica y en los dos primeros de la educación media, con lo que queda demostrado que la faja etaria que va de los 12 a los 16 años es las más problemática y donde se concentra el bullying homofóbico. Hostigamiento que es coincidente con otras formas de bullying profusamente descritos en la literatura especializada.

En esta misma línea el año 2011 el MINEDUC, en el contexto de la promulgación de la ley n. 20.536 sobre violencia escolar o también conocida como "Ley Antibullying", realizó una encuesta nacional con estudiantes de octavo básico (último año de la educación básica) con el propósito de conocer los alcances de las agresiones y acoso escolar en los establecimientos educacionales del país. El estudio llevado a cabo en 5.855 escuelas con una población encuestada de 228.883 en todas las regiones develó que las agresiones más frecuentes de acuerdo con una encuesta que contaba con 34 preguntas eran: los insultos, 69\%; las peleas, 50\%; rumores mal intencionados, 47\%; amenazas, 36\%; daños a la infraestructura, $28 \%$; robos o hurtos, 22\%; agresiones con arma blanca, $5 \%$; y agresiones con armas de fuego, $4 \%$.

Respecto de la dependencia administrativa de las escuelas el 38\% correspondía a escuelas municipalizadas, el $29 \%$ a escuelas particulares subvencionadas y el $17 \%$ a escuelas particulares pagadas. La distribución de las agresiones según nivel socioeconómico fue el siguiente: $23 \%$, bajo; $44 \%$, medio bajo; $34 \%$, medio; $22 \%$, medio alto; y $15 \%$, alto. Por regiones las agresiones alcanzaron los siguientes valores: Arica y Parinacota, 41\%; Tarapacá, 41\%; Antofagasta, 36\%; Atacama, 29\%; Coquimbo, 28\%; Valparaíso, 37\%; RM, 42\%; O’Higgins, 34\%; Maule, 20\%; Biobío, 31\%; La Araucanía, 16\%; Los Ríos, 23\%; Los Lagos, 26\%; Aysén, 27\%; y Magallanes, $21 \%$, siendo que el promedio nacional alcanzó el 32\%.

Con relación al acoso escolar, el $9 \%$ de los estudiantes reportó haber sido víctima de acoso escolar. Las conductas más recurrentes fueron el maltrato verbal con un $10 \%$, el maltrato social con un $9 \%$, el maltrato físico con un $5 \%$ y el ciberbullying con un 5\%. Respecto de la dependencia administrativa de los establecimientos el $10 \%$ correspondió a escuelas municipales, el $9 \%$ a subvencionadas y el $8 \%$ a particulares pagadas. La distribución del acoso escolar por nivel socioeconómico fue el siguiente: $11 \%$, bajo; $11 \%$, medio bajo; $8 \%$, medio; $8 \%$, medio alto; y $8 \%$, alto. Por regiones la distribución fue la siguiente: Arica y Parinacota, 10,6\%; Tarapacá, 
9,7\%; Antofagasta, 8,5\%; Atacama, 9,4\%; Coquimbo, 9,6\%; Valparaíso, 9,3\%; RM, 9,2\%; O'Higgins, 10,2\%; Maule, 9,5\%; Biobío, 9,4\%; La Araucanía, 8,4\%; Los Ríos, 8,9\%; Los Lagos, 8,4\%; Aysén, 9,5\%; y Magallanes, 8,3\%, siendo que el promedio nacional alcanzó el 9,2\% (MINEDUC, 2011).

Estas cifras reflejan la preocupación de las autoridades del MINEDUC por el tema, situación que queda demostrada tanto en la promulgación de una ley específica de violencia escolar como en el intento de dimensionar el problema a través de un estudio a nivel nacional. El haber focalizado el estudio en $8^{\circ}$ Básico demuestra que este tipo de violencia se tiende a concentrar en los últimos años de la educación básica y primeros de la enseñanza media.

Retomando el estudio en torno a las denuncias de discriminación por orientación sexual, más allá de las cifras, las quejas guardan relación con el malestar que ocasiona en los estudiantes el ser objeto de agresiones verbales, humillación pública y burlas motivada por una orientación sexual distinta a la heterosexual o presunción de ella; ya sea por parte de compañeros, o ya sea por parte de profesores u otros agentes educativos. Sin embargo, las quejas más frecuentes apuntan a los profesores, inclusive mucho más que a los pares. Numerosos relatos señalan que el acoso u hostigamiento de los compañeros deviene del maltrato verbal u humillación pública de un profesor.

Un ejemplo de ello son los interrogatorios, varios de ellos en presencia de otras personas, a que fueron sometidos ciertos estudiantes, en un intento de descubrir una supuesta homosexualidad. Es decir, se trata de una suerte de "hermeneútica de la sospecha", de acuerdo con la caracterización de Lee Edelman (2004), según la cual toda expresión o forma de sociabilidad sexuada es examinada exhaustivamente para verificar si oculta algún significado o trazo referido a la homosexualidad. De ser positiva la búsqueda, las conductas consideradas desviadas se procura normalizarlas, apelando inclusive a la violencia física.

En ese contexto de auténtica persecución de las sexualidades disidentes no son inusuales las "listas negras" en los colegios en un intento de saneamiento disciplinario de los sujetos indeseados cuya extirpación de la comunidad educativa se hace esgrimiendo la vieja consigna que señala que "una manzana podrida puede echar a perder todo el cajón", ya sea por el mal ejemplo para sus compañeros o, en el peor de los casos, porque pueden inducir a través de la seducción amorosa a otro incauto estudiantes. En el primero de los casos el 32,3\% de los denunciantes acusa haber sido expulsado o visto condicionada su matrícula por este motivo, y en el segundo el 3\%.

La situación se torna más compleja cuando el estudiante es acusado de tener expresiones erótico - afectivas públicas que subvierten el orden establecido y atentan contra una rígida moral heterosexista, como es, por ejemplo, darle un beso a un compañero o expresar la intención de mantener una relación amorosa homoerótica.

Las sanciones más usuales en las escuelas son la cancelación de matrícula, las expulsiones sumarias y la condicionalidad. La falta de un debido proceso $(4,6 \%)$ y la presencia de disposiciones abiertamente discriminatorias u homofóbicas presentes en los proyectos educativos institucionales y/o reglamentos de convivencia escolar, particularmente de colegios confesionales, son otros de los elementos que configuran el cuadro de denuncias. 
Asimismo, no deja de llamar la atención que muchas de las denuncias $(15,3 \%)$, subrayen que la sanción o medida disciplinaria adoptada por el establecimiento se debió simplemente al hecho que el estudiante no esconde su orientación sexual. Es decir, no hubo ningún acto reñido con la disciplina o trasgresión de las normas de convivencia escolar, solo asumir públicamente una orientación sexual o identidad genérica disidente. Con todo, esa actitud más decidida es abrumadoramente más común entre las jóvenes lesbianas que reivindican ellas mismas y sus familias su derecho a ser respetadas por su orientación sexual. Los varones, o más bien sus familias, por el contrario, al momento de formalizar la denuncian tienden a insistir que sus hijos fueron injustamente sancionados debido a que algún gesto, actitud o comportamiento fue mal interpretado por parte de sus profesores u otros agentes educativos; esto es, no solo no reivindican una identidad disidente, sino que terminan avalando con sus discursos el orden heteronormativo imperante en la escuela.

Por último, los efectos psicoemocionales en los estudiantes sancionados o acusados de homosexualismo se expresan, como se desprende de las propias denuncias, en depresiones, aislamiento e inclusive en intentos de suicidio (al menos en uno de los casos denunciados). En el ámbito pedagógico las consecuencias del hostigamiento homofóbico se expresan en descenso del rendimiento académico, descuido de los deberes escolares, no asistencia a clases y deserción del sistema escolar. Esta apreciación es coincidente con las descripciones de la literatura especializada referida al bullying, donde esas consecuencias suelen aparecer en un número importante de casos.

En el plano de la convivencia social la discriminación se expresa claramente a través del bullying homofóbico, contenido en maltrato verbal de parte de profesores y compañeros, segregación, humillaciones públicas y burlas. La exposición a través de las redes sociales, si bien mucho menos frecuente, es otro de los medios a través de los cuales se manifiesta la discriminación.

Algo parecido ocurre con los reglamentos de convivencia escolar o proyectos educativos institucionales, particularmente de colegios confesionales, que si bien poco significativos si considerada la totalidad de las denuncias ante el MINEUC, contienen definiciones homofóbicas o establecen sanciones o medidas abiertamente discriminatorias hacia estudiantes LGBT. Situación que contraviene las disposiciones del propio Ministerio, en el sentido de desincentivar toda medida discriminatoria de parte de los establecimientos educacionales, incluida la orientación sexual, con el propósito de promover escuelas inclusivas (MINEDUC, 2013).

Lo más preocupante en relación a este tipo de discriminación es que los planes y programas de educación sexual en Chile y las prácticas educativas, salvo contadas excepciones, han tendido a perpetuar o legitimar indirectamente estos comportamientos por la omisión o invisibilización del tema tanto del currículo como de la convivencia escolar cotidiana ((MINEDUC, 2003), por la asociación que aún se continúa haciendo entre vírus da imunodeficiência humana/ síndrome da imunodeficiência adquirida (VIH/SIDA) y homosexualidad, por el desinterés de parte de las entidades responsables de la formación de profesores de introducir la temática en las mallas de formación profesional, o simplemente por la evasión 
del tema a través de un eufemismo que lo describe como un "problema difícil de abordar" o un "nudo crítico" que en algún momento futuro (nunca bien definido) debe ser abordado (MINEDUC, 2005a, 2005b).

Los siete programas de educación vigentes, ${ }^{2}$ elaborados por universidades nacionales u organismos especializados en sexología o medicina reproductiva, en su mayoría, han puesto su acento en consideraciones biológico - reproductivas, prevención del embarazo o exhortaciones morales que apelan al desarrollo del amor heterosexual. La excepción es el Programa de Aprendizaje en Sexualidad y Afectividad (PASA) que no solo aborda el asunto, sino que sus gestores tienen una visión crítica respecto de los otros programas que insisten en calificar a la homosexualidad como un trastorno (Palma, Reyes y Moreno, 2013).

A este respecto cabe recordar que el programa de educación sexual de la Universidad San Sebastián fue cuestionado, precisamente, por este punto. Cuestionamiento que además de aparecer en la prensa (Emol, 6 de junio de 2012) obligó al Ministro de Educación de la época a dar explicaciones públicas: "Ese programa tiene un error y pediremos que se enmiende ese error. La homosexualidad y el lesbianismo no son un trastorno" (La Tercera, 6 de junio de 2012, "en línea”; El Dínamo, 6 de junio de 2012).

En la misma línea la directora del Programa Aprendiendo a Querer de la Universidad Católica de la Santísima Concepción sostiene que, siguiendo las enseñanzas de la Iglesia, condena la práctica homosexual, pero si encuentra personas homosexuales se las trata con la misma dignidad y se las puede ayudar a ver la inconveniencia de este tipo de práctica.

El problema de este discurso es que no se puede pretender acoger o mostrar una actitud comprensiva hacia las personas homosexuales negando o cercenando partes importantes de sus vidas. Aunque revestido de un ropaje comprensivo este tipo de discurso, en verdad, no resulta ser más que una flagrante violación de derechos humanos escudado en consideraciones morales o religiosas.

Igualmente, cuestionable resulta la actitud del MINEDUC, que, apelando a la libertad de elección de las familias de la educación de sus hijos o un pretendido pluralismo, terminan indirectamente avalando ideas, que aun cuando no emanaron de esa repartición pública, están en la base de la discriminación homofóbica o son articuladoras de las mismas (Palma, Reyes y Moreno, 2013).

Más allá de las controversias que se puedan suscitar acerca de los límites del Estado en materia de educación sexual o de la capacidad de las familias para

2 Los programas en cuestión son: Teen Star, de la Pontificia Universidad de Chile; Programa de Educación en Valores, Afectividad y Sexualidad (PAS), de la Universidad San Sebastián; Aprendiendo a Querer, de la Universidad Católica de la Santísima Concepción; Sexualidad, Autoestima y Prevención de Embarazos en Adolescentes, de Asociación Chilena de Protección de la Familia (APROFA); Adolescencia, Tiempo de Decisiones, de Centro de Medicina Reproductiva y Desarrollo Integral del Adolescente (CEMERA, Facultad de Medicina de la Universidad de Chile); Programa de Aprendizaje en Sexualidad y Afectividad (PASA), del Departamento de Psicología de la Universidad de Chile; y Curso de Educación Sexual Integral del Centro de Educación Integral del Dr. Capponi (CESI). 
proveer una educación oportuna, informada y con una base científica, es indudable que el sistema escolar chileno abriga una serie de manifestaciones homofóbicas expresadas en discriminación por orientación sexual, en bullying homofóbico o en violencia simbólica contenida en los discursos y en las prácticas de aula que es necesario revertir.

En los siguientes acápites se esbozan algunas pistas, a partir de las experiencias descritas anteriormente, para combatir situaciones discriminatorias fundadas en la orientación sexual, lidiar con estudiantes disidentes del orden heteronormativo, proveer estrategias de manejo del bullying homofóbico, pero sobre todo prevenir esas situaciones a través de una educación inclusiva, promoción de los derechos humanos de las personas LGBT, fomento de una cultura de la paz y gestión de espacios escolares seguros.

\section{URGENCIA DE MEDIDAS PREVENTIVAS}

En primer lugar, no se puede obviar que la discriminación por orientación sexual puede causar serios daños en la salud mental y psicológica de las personas que son objeto de discriminación, particularmente cuando ese rechazo e intolerancia tiene lugar en la niñez o en la adolescencia.

En el contexto escolar esa discriminación suele expresarse en distintas formas de violencia que van de las agresiones físicas a la exclusión social, pasando, por las descalificaciones, las burlas o los insultos. Comúnmente se denomina a ese fenómeno como "bullying homofóbico", por cuanto es un tipo específico de violencia escolar fundado en sentimientos de rechazo o repulsión hacia niños y jóvenes que son disidentes del orden heteronormativo o evidencian conductas o trazos de personalidad que disienten de los ideales de masculinidad o feminidad impuestos por ese mismo orden. Sentimientos que se inscriben y configuran lo que suele definirse como homofobia (Blumenfeld, 1992; Borrillo, 2001; Cornejo, 2010), la cual puede permear tanto la cultura y las instituciones, así como el accionar de las personas y cuyas expresiones se justifican y retroalimentan mutuamente.

A este respecto cabe recordar que numerosos estudios revelan una estrecha conexión entre bullying homofóbico reiterado en el tiempo en contextos escolares y ocurrencia de cuadros de depresión, ansiedad, pérdida de confianza, baja autoestima, retraimiento, marginación social, culpa y sueño alterado. Es decir, estudiantes objeto de bullying homofóbico en sus escuelas manifiestan con mayor frecuencia sentimientos autodestructivos, además de mayor probabilidad de suicidarse (UNESCO, 2012).

Otros efectos del bullying homofóbico es la mayor propensión de estos jóvenes, dado el clima de hostigamiento que los rodea, al uso de alcohol, drogas, así como la mayor exposición a conductas sexuales de riesgo. Sin contar en el plano académico el ausentismo escolar o bajo rendimiento. Cabe recordar que estas características son comunes a otras formas de bullying (American Educational Research Association, 2013).

Estos antecedentes son suficientes argumentos para que las escuelas y el sistema escolar en su conjunto diseñen y adopten una serie de estrategias preventivas 
de modo de desincentivar este tipo específico de violencia escolar, especialmente porque en Chile como en los países de la región el tema no es abordado.

La razón de ello es que no ha habido un reconocimiento de la especificidad del problema, del impacto que provoca el fenómeno en la psiquis de las víctimas, los agresores y testigo de los hechos de violencia, así como de sus efectos en los procesos de aprendizaje; motivo por el cual los sistemas educacionales deben enfrentar el bullying homofóbico, más allá de su aceptación o reparos frente a la homosexualidad, dado al impacto negativo de este tipo de violencia en el derecho a la educación.

A este respecto cabe señalar que cualquier proyecto preventivo debe partir de los derechos humanos y el derecho a acceso a la educación, libre de todo tipo de discriminación y violencia.

La insistencia en la promoción de los derechos humanos radica en que el bullying homofóbico o cualquier manifestación de discriminación por orientación sexual y/o identidad genérica es una amenaza al derecho a la educación expresado en los Objetivos de Desarrollo del Milenio y el Foro Marco de Acción de Dakar que establece la relación entre el derecho a la educación de calidad y el derecho a un ambiente de aprendizaje seguro y libre de violencia. En este sentido la homofobia en contexto escolar no solo vulnera esas dimensiones, sino los objetivos de la Educación para Todos relacionado con el acceso, la retención y éxito escolar.

Entre las múltiples medidas que se pueden implementar para combatir toda forma de discriminación fundada en la orientación sexual, identidad genérica o violencia homofóbica es clave contar con una política definida centrada en los derechos humanos, debido a las resistencias que puede generar el abordaje del tema en los propios profesores, motivada por apreciaciones personales moralizantes instigadas por ciertos formadores de opinión.

En países que carecen de políticas o ellas son insuficientes resulta fundamental establecer alianzas estratégicas con personas y agrupaciones interesadas en la defensa y promoción de derechos humanos, particularmente agrupaciones LGBT. Asimismo, se hace necesario trabajar con las autoridades de educación, líderes comunitarios, además de los medios de comunicación social de modo de convertirlos en aliados en la lucha contra toda forma de discriminación hacia esas personas.

Una política genérica contra la violencia escolar u otras políticas que aborden la discriminación por razones de raza, color, religión o sexo pueden constituirse en un punto de partida para enfrentar la discriminación por orientación sexual y el bullying homofóbico.

\section{ESTRATEGIAS DE PREVENCIÓN, MANEJO Y COMBATE DE LA VIOLENCIA HOMOFÓBICA ESCOLAR}

En el plano estrictamente educativo cualquier medida que se adopte debe tender no solo a una "tolerancia de lo inevitable" (Cornejo, 2010) o tolerancia pasiva de los estudiantes LGBT, disidentes del ideario heterosexista, sino adoptar una postura activa que desincentive el uso de la violencia en cualquiera de sus formas, como una actitud más propositiva que se anticipe a esos hechos. 
Así cualquier propuesta de manejo, prevención o combate debe partir de la promoción y defensa de los derechos humanos y como consecuencia de ello el incentivo de una cultura de la paz. Cultura que además de garantizar la igualdad y equidad permita el desarrollo armonioso e integral de todos sus miembros en espacios seguros.

No obstante, al ser el bullying en cualquiera de sus formas, y el homofóbico en particular, un problema más o menos generalizado en la mayor parte de las escuelas del mundo (UNESCO, 2012), no se puede desconocer que no existen recetas únicas de intervención, manejo o prevención que puedan funcionar de igual modo en todos los contextos educativos (Fante, 2012; Lopes, 2011). Y si bien todo proyecto debe partir de un principio universal básico, irrenunciable, cual es el respeto irrestricto de los derechos humanos, las estrategias o énfasis pueden variar de un contexto a otro.

Es decir, el núcleo central de todo proyecto deben ser los derechos humanos frente a los cuales cualquier otra consideración de orden filosófica, moral o religiosa debe estar supeditada, aún en aquellas comunidades educativas que se definen a partir de paradigmas que, aunque valiosos en muchos aspectos, eventualmente pueden incurrir en violaciones de derechos de personas o grupos.

La advertencia anterior no es antojadiza especialmente cuando se constata que los derechos de las personas disidentes sexuales, en muchos ambientes incluido el educativo, son vulneradas, relativizadas o puestas en dudas en virtud de apreciaciones, creencias o posturas que caben dentro del ámbito de lo privado y que en ningún caso se pueden imponer como norma de convivencia social, aun para los sujetos que adhieren a esas posturas o creencias. Dicho en otros términos, los derechos humanos de los disidentes sexuales que devienen de su propia orientación sexual y/o identidad genérica no puede cuestionarse, negarse, invisibilizarse o menos aún intentar modificarse escudados en creencias o valores particulares sin grave daño a la integridad psicoemocional del sujeto objeto de la agresión y de la convivencia social de toda una comunidad.

Como ejemplo que ilustra lo anterior, se pueden citar las denuncias de algunos colegios confesionales chilenos que en sus manuales de convivencia escolar contenían expresiones discriminatorias de las disidencias sexuales o abiertamente homofóbicas, contraviniendo las instrucciones del propio MINEDUC que desde hace algunos años ha insistido en la necesidad de erradicar de la documentación oficial de los establecimientos toda expresión o lenguaje que denote discriminación o induzcan a ella. Este tipo de denuncias está registrado tanto en el listado de denuncias de discriminación por orientación sexual del Ministerio, como las realizadas por el MOVILH a través de la prensa.

Clave en el diseño, elaboración e implementación de todo proyecto de manejo o prevención de la violencia homofóbica o antidiscriminatorio es el aporte y coordinación de todos los estamentos que componen las comunidades educativas. La omisión de cualquiera de ellos no solo resta fuerza y legitimidad al proyecto, sino que limita sus posibilidades de éxito.

A continuación, se describen algunas pistas agrupadas en diez ejes que pueden ayudar al esbozo de proyectos de manejo y combate de la violencia homofóbica y 
prevención de toda forma de discriminación fundada en la orientación sexual y/o identidad genérica. ${ }^{3}$

\section{PROMOCIÓN DE DERECHOS HUMANOS}

El principio básico sobre el cual se debe construir todo proyecto educativo antidiscriminatorio son los derechos humanos, motivo por el cual no se puede permitir ninguna acción de contenido homofóbico (al igual que sexista, racista, xenófobo, etc.) que atente contra la libertad de expresión de personas o grupos.

Es decir, no se puede pretender disciplinar, asimilar, omitir o negar la orientación sexual y/o identidad genérica de una persona o un grupo de ellas bajo el pretexto de no agresión a las creencias, valores o posturas de los otros estudiantes; o aún para salvaguardar la integridad de los propios disidentes. El intento de omisión e invisibilización de estos estudiantes es ya una agresión que no puede ser tolerada.

Las implicancias prácticas de este principio son las que resultan más difíciles de cumplir para algunos establecimientos, especialmente de cuño religioso; pues, no solo se resisten a eliminar expresiones homofóbicas de sus documentos oficiales, sino que coartan (so pena de expulsión) la posibilidad de que los jóvenes disidentes expongan abiertamente su orientación sexual o expresen públicamente sus afectos.

El argumento recurrente es el mal ejemplo que esas conductas podrían ocasionar en los estudiantes de los cursos inferiores o el reclamo u oposición de los padres de los otros estudiantes.

\section{HOMOFOBIA ESCOLAR EN LA MIRA}

En consonancia con el punto anterior no se puede tolerar el uso de lenguaje homofóbico o discriminatorio de ningún miembro de la comunidad educativa. Esta medida implica erradicar todo tipo de comentarios, insultos o bromas de contenido homofóbico de los espacios escolares.

Este tipo de violencia está alojada en la propia cultura que legitima ciertas prácticas discursivas que se proyectan en la escuela, motivo por el cual una de las funciones pedagógicas fundamentales en la lucha contra la homofobia consista en no bajarle el perfil a esas prácticas y no aceptarlas bajo ningún punto de vista.

Cabe recordar que una de las quejas más recurrentes contenidas en el catastro de denuncias del MINEDUC apunta, precisamente, al uso de un cierto lenguaje burlesco, despectivo, humillante o discriminatorio, no solo de parte de los pares de la víctima, sino de sus propios profesores. Es más, de acuerdo con los relatos contenidos en ese catastro la mayor parte de las veces el bullying homofóbico de los pares

3 Algunos insumos adicionales que pueden ayudar al diseño de los planes preventivos o de manejo de la violencia homofóbica se encuentran en el texto de UNESCO (2012), Respuestas del sector educación frente al bullying homofóbico; MOVILH (2010), Educando en la diversidad: orientación sexual e identidad de género en las aulas; Colectivo de Lesbianas, Gays, Transexuales y Bisexuales de Madrid (COGAM) (2005), Homofobia en el sistema educativo, o en el material educativo de la ONG "Todo mejora" (www.todomejora.org). 
se desencadena a partir de una humillación pública o uso de lenguaje homofóbico ofensivo de parte de un profesor u otro agente educativo.

Otro aspecto para tener en consideración en el combate de la homofobia en el contexto escolar es la necesidad de desincentivar el uso de estereotipos de género con relación a las personas LGBT. Nuevamente, de acuerdo con la información recabada a través del MINEDUC, la prensa o entrevistas a víctimas y testigos de bullying homofóbico se constata que una de las causales de discriminación o violencia homofóbica es el recurso a ciertos estereotipos de género, promovidos en múltiples ocasiones por los propios establecimientos educacionales, que caricaturizan lo que se espera sea el comportamiento socialmente aceptado de un hombre o una mujer.

De allí, que pese a las prohibiciones del MINEDUC, sean relativamente frecuentes las expulsiones sumarias de estudiantes que no se encuadran a los padrones de género esperados o por expresiones afectivas en público. Para no aparecer a los ojos de las autoridades ministeriales como entidades discriminadoras, los establecimientos suelen convencer a los padres de los estudiantes acusados de conductas "impropias" de retirar a sus hijos diciéndoles que esas comunidades no son apropiadas para ellos. De ese modo, la expulsión se esconde, apareciendo como una decisión del apoderado cuando en verdad era una decisión ya resuelta por las autoridades del establecimiento.

Otra práctica habitual es la aceptación condicionada de los disidentes, es decir, se los acepta en los colegios siempre y cuando no expongan públicamente su orientación o identidad genérica, ni mucho menos exterioricen sus inclinaciones afectivas; esgrimiendo que esas conductas podrían confundir a sus compañeros de cursos menores o para evitar el reclamo de otros apoderados. En otras palabras, los colegios promueven la invisibilización de los disidentes, pues, su exposición pública ofende y sus afectos violentan a la comunidad escolar. En el mejor de los casos, eufemísticamente, se argumenta que esas medidas son para "proteger" a esos estudiantes de las agresiones, cuando lo correcto sería esforzarse a través de iniciativas formativas en erradicar toda forma de homofobia y exclusión de las comunidades escolares.

\section{TOMA DE CONCIENCIA}

Cualquier diseño, elaboración o puesta en práctica de un plan preventivo, de manejo o combate de la violencia homofóbica debe tener como punto de partida la concientización de las comunidades educativas acerca del problema y su compromiso con las estrategias diseñadas.

No obstante, esa toma de conciencia no puede ser un obstáculo o una fase previa para la implementación de cualquier proyecto preventivo o de manejo. La toma de conciencia y la practica son procesos que se retroalimentan y desarrollan simultáneamente. Nadie puede pretender luchar contra la homofobia solo desde la teoría. La toma de conciencia se plasma y concretiza a partir de las prácticas cotidianas.

Este punto es seguramente uno de los más difíciles de llevar a la práctica, pues, de acuerdo con las entrevistas a distintos agentes educativos, ningún establecimiento se reconoce como homofóbico o discriminatorio. Todos ellos apuestan por la integración de todos sus estudiantes. El problema se presenta cuando los 
estudiantes disidentes sexuales exteriorizan su orientación sexual o hacen público sus afectos. Es decir, en el imaginario de estos educadores, se puede ser homosexual en cuanto no se verbalice o se pretenda exponer dicha orientación, imponiéndoles una verdadera ley del silencio.

En vista de lo anterior, nuevamente en la toma de conciencia el elemento fundamental debe ser el respeto y promoción de los derechos humanos, no desde una perspectiva paternalista que concibe a los sujetos LGBT desde la subalternidad o la incapacidad para ejercer sus derechos en plenitud, sino desde una relación simétrica entre todas las sexualidades, motivo por el cual tanto la jerarquización de las sexualidades (Borrillo, 2001) como la pretensión de conceder derechos desiguales a disidentes, si comparados con los que gozan los heterosexuales, resulta contraproducente y atentatorio al principio de equidad e igualdad que subyace a todo derecho humano.

\section{FORMACIÓN DE PROFESORES}

Durante mucho tiempo la literatura referida a bullying u otras formas de violencia escolar ignoró la influencia del género y sexualidad. Esta afirmación pareciera quedar refrendada por los testimonios de los agentes educativos entrevistados, que normalmente desconocen o no han tomado conciencia que el bullying puede tener un importante componente homofóbico.

Probablemente, este descuido se explique por la homofobia cultural imperante que tiende a bajarle el perfil a este tipo de violencia en contextos escolares o a suponer que esas agresiones son apenas bromas entre adolescentes. Esta apreciación es coincidente con el estudio de la American Educational Research Association (2013), que sostiene que los profesores, en general, parecen menos dispuestos a intervenir en situaciones de bullying u hostigamiento homofóbico en las escuelas.

No se puede obviar tampoco, que en general los profesores en Chile (situación probablemente similar en otras latitudes) no poseen las competencias y habilidades, dadas las falencias en su formación profesional, para abordar temáticas relativas a educación sexual en los colegios y de modo especial todo lo referido a diversidad sexual. Usualmente el tratamiento del tema subraya los aspectos biológicos - reproductivistas y una concepción binaria de la sexualidad que solo da espacio a las relaciones heterosexuales, invisibilizando u omitiendo toda posibilidad de realización erótico - afectiva que escape o sea disidente de ese binarismo (Cornejo, 2008).

En este sentido los propios docentes entrevistados sostienen que, en general, los profesores no solo carecen de las competencias para abordar temáticas relativas a educación sexual, sino también para lidiar con situaciones problemáticas en que se vean envueltos estudiantes LGBT.

Esa incapacidad se explica según ellos mismos, por el descuido de las universidades formadoras de profesores que no incluyen, o lo hacen muy superficialmente, estas temáticas en las mallas curriculares. De allí, que resulte prioritario proporcionar formación a los futuros profesionales de la educación, tanto en educación sexual, valores, derechos humanos y habilidades para el abordaje y manejo del bullying homofóbico o cualquier otra conducta disruptiva que comprometa la convivencia escolar o sea motivo de discriminación. 
Esa formación debe estar en sintonía con la que debe recibir el resto de la comunidad educativa, cuyos acentos están puestos en el respeto, el conocimiento y las habilidades necesarias para trabajar con realidades diversas en lo que a sexualidad y género se refiere; además de las distintas formas de familia.

\section{PROGRAMAS DE EDUCACIÓN SEXUAL}

Uno de los temas claves en la eliminación de la discriminación basada en la orientación sexual y/o identidades genéricas, motivada por el desconocimiento, ignorancia o ideas equivocadas con relación a la diversidad sexual es el fomento, desarrollo e implementación de planes y programas de educación sexual. Programas cuyo acento y enfoque debe estar puesto en los derechos humanos.

Incluir la educación sexual en los currículos escolares implica presentar la diversidad de orientaciones sexuales e identidades genéricas, expresadas no de manera jerárquica. Es decir, el discurso no debe dar por descontado que va dirigido a una población exclusivamente heterosexual, y contemplar que existen otras orientaciones e identidades igualmente legítimas y valiosas.

Esto supone también ofrecer en las distintas asignaturas referentes que muestren la diversidad de orientaciones sexuales, identidades genéricas y variedades de familias, entre las que se hallan las homoparentales.

Todo este esfuerzo innovador necesariamente debe estar en sintonía con la formación de los futuros profesores y las prácticas de aula, pues, no basta con tener programas de educación sexual inclusivos desde la perspectiva de la diversidad sexual e inspirada en los derechos humanos, sino también contar con profesores capaces de ponerlos en práctica.

No se puede obviar tampoco, a partir de la experiencia chilena, que ha habido descuido por la educación sexual, por cuanto en varios momentos en las décadas pasadas no hubo programas de educación sexual o estos evidenciaban un marcado sesgo moralizante que legitimaba la exclusión de los disidentes sexuales tras un lenguaje supuestamente acogedor e inspirado en el amor, pero limitado al amor heterosexual y con exclusión de cualquier otra expresión amorosa (Cornejo, 2008).

De allí, que el desafío sea doble, pues, se requiere no solo de programas inclusivos carentes de todo sesgo moralizante y excluyente, sino que además los profesores cuenten con las competencias necesarias para su implementación, sensibles a la diversidad y atentos a todo intento manipulador.

\section{APORTES DE LA SOCIEDAD CIVIL}

Identificar a los potenciales aliados, sin duda, puede ayudar en la implementación de las intervenciones pedagógicas tendientes a erradicar toda forma de exclusión o violencia homofóbica. El recurrir a entidades estatales u organizaciones no gubernamentales especializadas en derechos humanos, particularmente de personas LGBT, puede ser de gran ayuda tanto para el diseño de programas educativos como en la ejecución de planes antidiscriminatorios y preventivos del bullying homofóbico. 
Establecer acciones de trabajo conjunto con asociaciones de madres y padres para conseguir reducir los problemas de aceptación de los hijos e hijas LGBT al interior de sus familias y de las escuelas resulta crucial. No se puede desconocer que uno de los mayores problemas de aceptación que enfrentan los jóvenes disidentes sexuales es, precisamente, con sus familias.

No son pocos los estudiantes, como se desprende de los testimonios de los entrevistados, que temen contar en sus casas que son acosados u objeto de bullying homofóbico por parte de sus compañeros, ya sea por vergüenza, o ya sea porque están convencidos de que serán castigados por sus padres. Esa convicción los lleva a ocultar a sus familias la violencia que están sufriendo en la escuela, con el consecuente daño psicoemocional que normalmente se traduce en depresión y retraimiento.

A diferencia de otros tipos de bullying en que las familias solidarizan con el menor agredido, en el caso de bullying homofóbico se produce una doble victimización, por cuanto ese menor o adolescente es violentado tanto por sus pares como por sus familias, motivo por el cual la colaboración que puedan prestar agrupaciones de padres/madres de hijos LGBT puede ser de gran ayuda, tanto en la aceptación incondicionada de sus hijos, como en la comprensión de los procesos de desarrollo psicoemocional y afectivo que están viviendo sus hijos.

Así, fomentar la cooperación con asociaciones LGTB o de padres/madres de hijos LGBT resulta fundamental, especialmente por el apoyo que estas organizaciones pueden prestar en las actividades escolares; además de constituirse en referentes positivos tanto para los mismos jóvenes como sus familias.

En el caso chileno esta alianza es fundamental, no solo por los referentes positivos que se pueden encontrar en estas organizaciones, sino porque la mayor parte de las iniciativas de colocar el tema de la diversidad sexual en la mesa de discusión en el ámbito educativo han sido impulsadas desde estas organizaciones. Es decir, ha sido la presión de la sociedad civil la que ha inducido a los agentes del Estado, especialmente del MINEDUC, a incursionar en el tema.

\section{CONTENCIÓN, SEGUIMIENTO Y TUTORÍAS}

Un aspecto primordial en el manejo de la violencia homofóbica es el establecimiento de protocolos y mecanismos confidenciales para informar, atender y responder a los incidentes de bullying homofóbico que tienen lugar al interior de la escuela, proporcionando apoyo a los estudiantes violentados, a los agresores y a quienes son testigos de esos hechos.

En concordancia con lo anterior se hace necesario institucionalizar servicios de apoyo psicológico, de contención emocional, seguimiento y tutorías para los estudiantes que son víctimas de discriminación o bullying homofóbico. Esta labor se puede ver reforzada positivamente con la creación en los centros educativos de grupos de pares LGBT, cuidando si de no invisibilizar a sus participantes de las actividades e iniciativas del resto de la comunidad educativa o ser una excusa para su gettización.

Esta iniciativa es una tarea pendiente, razón por la cual es urgente constituir redes articuladas de contención y apoyo para niños y jóvenes víctimas de la violencia homofóbica en las escuelas; pues, las respuestas hasta ahora dadas a esta 
problemática, además de desarticuladas no son llevadas a cabo por especialistas. Esto es, se requiere de profesionales que no solo conozcan el tema, sino que ellos mismos se hayan desprendido de sus propios prejuicios y temores a la diversidad.

\section{MATERIAL EDUCATIVO}

Una de las quejas permanentes de los profesores es la falta de material educativo referido a la diversidad sexual. Probablemente este sea uno de los desafíos inmediatos en materia de educación sexual, pues, si bien se constata una creciente producción intelectual en torno al tema en el continente en la última década, aún resultan insuficientes en el plano educacional. Así, dotar a las bibliotecas escolares y centros de formación profesional de materiales (libros, vídeos, CD-ROMS, etc.) acerca de la diversidad sexual, que sirvan a la vez de recursos para el profesorado y de referencia para los estudiantes, se ha convertido en una necesidad y un complemento a la formación.

Un especial cuidado se ha de tener en la producción del material educativo referido a diversidad sexual, de modo de retirar todo elemento que refuerce los prejuicios y estereotipos hacia las personas LGBT.

En el ámbito nacional el tema es preocupante no solo porque aún son relativamente escasas las publicaciones referidas a diversidad sexual presentes en las bibliotecas del país, aún las producidas en otras latitudes, sino porque en el contexto educativo tales materiales son aún más escasos, ya sea porque la producción intelectual chilena es ínfima, o ya sea porque en los propios programas de educación sexual el tema no es abordado, salvo las alusiones condenatorias de la homosexualidad presentes en dos programas de circulación nacional.

La excepción la constituye el PASA del Departamento de Psicología de la Universidad de Chile, que dedica uno de sus capítulos al tema poniendo el acento en la capacidad de discernimiento y decisión de los propios jóvenes, el programa de UNESCO (2012), Respuestas del sector educación frente al bullying homofóbico, y las dos producciones del MOVILH cuyo propósito eran sensibilizar a la población respecto del tema.

Las grandes ausentes del debate han sido las facultades de educación de las distintas universidades del país, responsables de la formación de los futuros profesores. Sin lugar a duda una de las tareas pendientes de este sector es abordar este tema, así como todos aquellos tópicos que buscan ampliar los horizontes de la inclusión educativa, de modo de pasar de una educación centrada en las necesidades educativas especiales a una educación efectivamente inclusiva que considere a todos los grupos o personas objetos de exclusión de los sistemas escolares formales, incluidos los niños y jóvenes LGBT disidentes sexuales.

\section{ESCUELA ESPACIO SEGURO}

La literatura especializada tradicionalmente ha definido a la escuela como un espacio de seguridad en el cual los estudiantes deberían desarrollarse integralmente. 
No obstante, dados los hechos de violencia cada vez más recurrentes, ese espacio se ha convertido en una amenaza para algunos estudiantes, debido a normas rígidas con relación al género y sexualidad o lo desafiante que pueden resultar ciertos jóvenes disidente sexuales, que, a los ojos de sus pares, no solo no se ajustan a esas normas, sino que las desafían (Russell, 2011).

De allí que una de las tareas básicas en la lucha contra la violencia homofóbica sea la identificación de las áreas inseguras al interior de la escuela (pasillos, instalaciones deportivas, patios, inmediaciones de los establecimientos, etc.), de modo de buscar mecanismos que transformen esos espacios en ambientes seguros para toda la comunidad escolar. Esta iniciativa es coincidente con la información proveniente del registro de denuncias del MINEDUC y de los reportes de prensa; pues, en la casi totalidad de los hechos denunciados las agresiones tienen lugar en esos ambientes. Prácticamente todos los estudiantes denunciantes señalan a la escuela no sólo como un espacio inseguro donde se pone en riesgo su integridad física y psicológica, sino como un espacio poco gratificante para el aprendizaje.

Otra medida complementaria que puede ser de gran ayuda es ofrecer espacios o recursos para que los estudiantes LGTB o hijos de familias homoparentales puedan desarrollar sus habilidades de socialización e intercambio de experiencias con iguales.

\section{REGISTRO DE INFORMACIÓN}

Recopilar las evidencias que muestren como la discriminación y el bullying homofóbico se ha diseminado en las escuelas es otro de los ejes sobre los cuales se debe construir una política de manejo antidiscriminatoria.

Contar con esta información permite no sólo dimensionar el problema, al identificar a los grupos e individuos vulnerables, así como las distintas formas de acoso u hostigamiento, sino evaluar el impacto de las políticas implementadas, de forma tal, de hacer un seguimiento de las mismas e introducir las acciones correctivas si fuese necesario.

Probablemente uno de los ámbitos donde más se ha avanzado en la última década, es en el registro de las situaciones de discriminación por orientación sexual llevadas a cabo por el MINEDUC en todo el país. Sin embargo, el aspecto aún deficitario es el seguimiento de esas mismas denuncias. De acuerdo con los antecedentes del propio Ministerio la casi totalidad de ellas no derivan en medidas efectivas tanto por la demora en la resolución de los casos, como por lo excesivamente burocrático del sistema implementado que obliga a los denunciantes a ratificar más de una vez las denuncias a través de distintos canales.

En la práctica las pocas veces que se llega a una resolución, los estudiantes implicados en el hecho denunciado se han cambiado de establecimiento o ya han terminado sus estudios. La mayor parte de las veces los casos se cierran, a criterio de los funcionarios del Ministerio, porque los denunciantes no perseveran en la denuncia. Asimismo, las medidas de combate o manejo de la violencia homofóbica promovidas por el MINEDUC son más bien consultas y exhortaciones a los establecimientos. Poco hay en ellas de formativo o incentivo a las comunidades educativas para la creación de programas preventivos. 


\section{COMBATE A LA HEGEMONÍA DEL PARADIGMA HETEROSEXISTA}

Todas las medidas antes enunciadas son las condiciones mínimas de respeto y tolerancia hacia los estudiantes disidentes del ideario heterosexista. La ausencia u omisión de cualquiera de ellas u otras que se puedan implementar en el futuro, además de una flagrante violación de derechos humanos de los adolescentes y jóvenes LGBT, inviabilizan cualquier iniciativa tendiente a erradicar la discriminación y violencia homofóbica del espacio escolar.

Con todo, sería iluso pensar que estas medidas por sí solas resolverán el tema de la discriminación, la intolerancia o violencia hacia estos(as) estudiantes. La homofobia cultural (Blumenfeld, 1992; Borrillo, 2001), no solo configura y modela las mentalidades de las personas y los grupos sociales, sino que atraviesa las instituciones, entre ellas la escuela.

La homofobia ha adoptado diversas formas y tiene muchos orígenes. Inventada, fomentada y apoyada a través del tiempo por diferentes agencias de la sociedad - la religión, el gobierno, la ley y la ciencia - tiende a hacer erupción con un veneno especial cuando las personas imaginan una amenaza para la seguridad de los papeles que representan los géneros, de la doctrina religiosa o del Estado y la sociedad, o para la seguridad y la salud sexual del individuo. (Fone, 2000, p. 22)

Además, la homofobia alcanza a los propios sujetos objeto de la exclusión, a través del desprecio de sí mismo y de los otros ("homofobia internalizada"). Mecanismos como el rechazo, las amenazas, la vergüenza empujan a los disidentes sexuales a esconderse, a hacerse invisibles. "La invisibilidad - estigma que sufren los homosexuales es de otro tipo: es la de las identidades oprimidas, inhibidas, impensables [...]" (Tin, 2012, p. 474).

Tales sentimientos, indudablemente, inciden en el deterioro de la imagen personal, la autoestima y, eventualmente, en trastornos emocionales que en las situaciones más extremas pueden derivar en aislamiento, conductas antisociales y aún intentos de suicidio.

A este respecto cabe recordar que una de las quejas recurrentes de los disidentes sexuales, como se desprende de sus relatos autobiográficos y denuncias de discriminación ante el MINEDUC, es no solo la invisibilización de sus inclinaciones erótico - afectivas, sino también la omisión de su orientación sexual. La estructura escolar, aún para aquellos estudiantes que han "salido del closet", continúa con sus discursos y prácticas heterosexistas, haciendo de cuentas de cuentas que todos(as) son o deberían ser heterosexuales (Rich, 2010).

Esta actitud discriminatoria contenida en la omisión e invisibilización de los sujetos disidentes, se complementa con una suerte de "tolerancia de lo inevitable". Es decir, dado que formalmente no se puede expulsar a un estudiante del sistema escolar por su orientación sexual y/o identidad de género simplemente se lo tolera, pero sin que ello suponga aceptación o valoración. 
Estos antecedentes dejan en evidencia que medidas que se limitan a fomentar una tolerancia negativa no sólo no son educativas, sino que perpetúan un modelo inequitativo, injusto y discriminatorio, basado en el heterosexismo hegemónico y excluyente, que condena, persigue y margina a todo aquel que intente subvertir ese orden, denominado comúnmente como homofobia (Borrillo, 2001; Fone, 2000; Tin, 2012).

Así aceptar tiene poco que ver con la resignación o el conformismo, aspirando más bien a un nuevo orden donde las personas no son estigmatizadas o excluidas por su origen social, nivel educacional, pertenencia étnica, orientación sexual, identidad de género u otra condición. Aceptar supone valorar la diferencia como una fuente de riqueza y una oportunidad.

No obstante, para que ello sea posible no basta con la solidaridad nominal, el paternalismo o una aceptación que apenas esconde actitudes discriminatorias. Aceptar la diversidad sexual y las identidades genéricas, sin ambages o exclusiones, supone obligatoriamente revisar el paradigma heterosexista según el cual se modela la sociedad, se establecen las jerarquías y se configuran las personalidades, y cuya consecuencia inmediata es la homofobia que contamina y distorsiona las relaciones interpersonales.

Limitarse a medidas reactivas paliativas es no subrayar la dignidad humana. Es simplemente administrar el problema sin apuntar a las razones de fondo que lo explican y motivan, al punto de tornarse cómplice de un orden deshumano.

En el contexto educativo ese imperativo supone el compromiso activo de todos los miembros de las comunidades educativas con el quiebre del paradigma heterosexista, de modo de no solo romper con las lógicas formales de la exclusión, sino prestar atención a los rostros concretos de las víctimas; además de ser fieles a la vocación última de la escuela, cual es ser un espacio de acogida, seguridad, crecimiento, pero sobre todo humanización.

\section{CONCLUSIONES}

A modo de conclusión es necesario reiterar que el tema de la diversidad sexual es un aspecto aún pendiente en el sistema escolar chileno y, probablemente, en otros sistemas de la región dadas las resistencias a incorporarlo en el currículo escolar, particularmente en los planes y programas de educación sexual, pero también como tópico de reflexión y discernimiento en las comunidades escolares.

La práctica más habitual ha sido la negación, omisión, invisibilización o silenciamiento de las disidencias del orden heterosexista. Situación que, por cierto, además de no ser casual es una clara expresión de la violencia homofóbica imperante en la escuela (Dinis, 2011); pues, no solo hay violencia cuando se infringe daño de modo directo, sino también cuando se invisibiliza o cuando se pretende corregir un supuesto desvío.

Desde esta perspectiva las tareas más urgentes son, por una parte, insistir en la necesidad de programas de educación sexual que aborden la temática de la diversidad desde un enfoque de los derechos humanos, privilegiando el reconocimiento y valoración de la diversidad sexual y genérica y, por otra, una revisión profunda 
de los planes de formación de profesores, con el propósito de proporcionarles a esos futuros profesionales, por una parte, las competencias y habilidades necesarias para asumir los desafíos y complejidades que impone la sociedad contemporánea, caracterizada por el pluralismo, las disidencias y el multiculturalismo y, por otra, el manejo de situaciones conflictivas motivadas por la homofobia y las relaciones interpersonales con estudiantes LGBT.

Con todo, no se puede soslayar que el profesor no solo es portador de los conocimientos que le provee la universidad, sino también de su propio acervo cultural familiar nutrido de saberes, valores, representaciones y cosmovisiones que terminan impactando su quehacer en el aula. Saberes que muchas veces son portadores de sexismo, machismo, homofobia, etc.

Si a esto sumamos una deficitaria u omisa formación académica respecto de determinados temas, las posibilidades de quiebre de los círculos de la discriminación y exclusión se tornan más difíciles, especialmente cuando esos profesores no solo son funcionales al orden heteronormativo, sino que agentes activos transmisores del mismo.

En esta misma línea, cabe recordar que las políticas hasta ahora implementadas en el ámbito escolar chileno básicamente han tendido a la integración, como ocurre en general con todas las iniciativas de equidad e igualdad de género (MINEDUC, 2010). Es decir, se ha incentivado una aceptación pasiva, pero en ningún caso ha habido reconocimiento y menos aún valoración de la diversidad sexual y/o genérica.

Sin desconocer la importancia de los elementos antes descritos, esto es, un currículo inclusivo, una formación profesional acorde a los requerimientos de una sociedad diversa, y de políticas que no aspiren solo a la integración, es preciso subrayar la centralidad de las comunidades educativas en tanto agentes propulsoras del cambio; pues, son ellas los entornos privilegiados donde se vivencia y se tornan posibles los proyectos inclusivos.

Sin embargo, para alcanzar ese objetivo es imprescindible que las comunidades escolares formalicen instancias de reflexión y discernimiento e implementen políticas y estrategias pedagógicas inclusivas, de modo de pasar de la aceptación pasiva ("tolerancia de lo inevitable") de los disidentes a una auténtica inclusión. En este sentido, el artículo más que proponer recetas únicas para llevar a cabo esta tarea sugiere ejes de reflexión o aspectos a tener en consideración a la hora de desarrollar tanto planes de prevención, manejo y combate del bullying homofóbico como proyectos inclusivos que no se agoten en acciones remediales reactivas, sino que aspiren al quiebre del paradigma heterosexista generador de la exclusión.

En otras palabras, los ejes propuestos, comunes a muchas experiencias inclusivas a nivel internacional, buscan ser un subsidio o un incentivo para las comunidades escolares, de modo que, reconociendo sus culturas locales, construyan sus propias propuestas de acuerdo a sus necesidades, acentos y peculiaridades, no olvidando que la lucha contra la homofobia o cualquier otra forma de exclusión es permanente y que cualquier programa que no busque romper con el paradigma heterosexista no puede ser calificado como un auténtico proyecto inclusivo de la diversidad. 


\section{REFERENCIAS}

American Educational Research Association. Prevention of bullying in school, colleges and universities. Washington, DC: American Educational Research Association, 2013.

Aprofa Capacitaciones - Asociación Chilena de Protección de la Familia. Sexualidad, Autorestima y Prevención de Emabarazos en Adolescentes. Santiago: APROFA, 2012.

Artiles, A.; Kozleski, E. Educación inclusiva en el siglo XXI: notas para un programa de investigación histórico-cultural. In: Cardona, M. C.; Chiner, E. (Eds.). Investigación educativa en escenarios diversos, plurales y globales. Alicante: AIDIPE; Universidad de Alicante, 2014. p. 51-65.

Blumenfeld, W. Homophobia: how we all pay the price. Boston: Beacon Press, 1992.

Borrillo, D. Homofobia. Barcelona: Ediciones Ballaterra, 2001.

Butler, J. Cuerpos que importan. Sobre los limites materiales y discursivos del sexo. Buenos Aires: Paidós, 2002.

Cemera - Centro de Medicina Reproductiva y Desarrollo Integral del Adolescente. Facultad de Medicina Universidad de Chile. Adolescencia, tiempo de decisiones. Santiago: CEMERA, 2012.

CEsi - Centro de Educación Sexual Integral Dr. CApponi. Curso de educación sexual integral. Santiago: CESI, 2012.

Cogam - Colectivo de Lesbianas, Gays, Transexuales y Bisexuales de Madrid. Comisión de Educación. Homofobia en el sistema educativo. Madrid: COGAM, 2005.

Cornejo, J. Ideas y representaciones de la homosexualidad en el contexto de los planes y programas de educación sexual en Chile. 2008. 367f. Tesis (Magíster en Educación) USACH, Santiago, 2008.

. Jóvenes en la encrucijada. Revista Última Década, Valparaíso: Universidad de Valparaíso, n. 32, p. 173-189, jul. 2010.

Dinis, N. Homofobia e educação: quando a omissão também é signo de violencia. Educar em Revista, Curitiba: UFPR, n. 3, p. 39-50, 2011.

Edelman, L. No future. Queer Theory and the Death Drive. Durham, NC: Duke University Press, 2004.

Emol. Revelan que plan del Ministerio de Educación tilda la homosexualidad como “trastorno".Emol, Santiago: E1 Mercurio, 6 jun.2012. Disponible en: <http://www.emol. com/noticias/nacional/2012/06/06/544193/revelan-que-plande-educacion-sexual-delmineduc-tilda-la-homosexualidad-como-trastorno.html>. Acceso en: 15 sept. 2014.

Fante, C. Cómo entender y detener el bullying y cyberbullying en la escuela. Bogotá: Actualización Pedagógica Magisterio, 2012.

Fone, B. Homofobia una historia. México: Editorial Océano, 2000.

La Tercera. Ministro Beyer admite "error" en programas escolares que catalogan la homosexualidad como "trastorno". La Tercera, Santiago, 6 jun. 2012. Disponible en: <http://www.latercera.com/noticia/educacion/2012/06/657-464922-9-ministro-beyeradmiteerror-en-programas-escolares-que-catalogan-la.shtml>. Acceso en: 10 sept. 2014. 
Lopes, A. Bullying. Saber identificar e cómo prevenir. São Paulo: Editora Brasiliense, 2011. Mineduc - Ministerio de Educación. Politica de educación en sexualidad. Para el mejoramiento de la calidad de la educación. 5. ed. Santiago: Gobierno de Chile, 2003.

Plan de Educación en Sexualidad y Afectividad. Santiago: MINEDUC, 2005a. Disponible en: <http://www.movilh.cl/documentacion/documentos/ PlanEducacionSexual.pdf $>$. Acceso en: 15 jul. 2014.

. Plan de Educación en Sexualidad y Afectividad (2005-2006). Santiago: Gobierno de Chile, 2005b.

. Orientaciones 2010-2011 con enfoque de género para la asesoría técnica ministerial en los territorios. Santiago: MINEDUC, 2010. Disponible en: <http://www.pasa.cl/ wp-content/uploads/2011/08/EnfoqueGeneroATP.pdf>. Acceso en: 30 sept. 2014.

. Encuesta nacional prevención, agresión y acoso escolar. Santiago: MINEDUC, 2011. Disponible en: <http://portales.mineduc.cl/usuarios/mineduc/doc/201207301558020. Encuesta_nacional_prevencion_agresion_acosoescolar_2011.pdf >.Acceso en: 30 sept. 2014.

. Discriminación en el contexto escolar. Orientaciones para promover una escuela inclusiva. Santiago: MINEDUC, 2013.

Movilh - Movimiento de Liberación e Integración Homosexual. Educando en la diversidad: orientación sexual e identidad de género en las aulas. Santiago: MOVILH; Universidad Diego Portales, 2010. Disponible en: <www.movilh.cl/documentacion/ educando-en-la-diversidad $>$. Acceso en: 20 feb. 2016.

. Nicolás tiene dos papás. Santiago: MOVILH, 2014. Disponible en: <www. movilh.cl/documentacion/nicolas-tiene-dos-papas>. Acceso en: 20 feb. 2016.

Palma, I.; Reyes, D.; Moreno, C. Educación sexual en Chile. Pluralismo y libertad de elección que esconde una propuesta gubernamental conservadora. Revista Docencia, Santiago: Colegio de Profesores, n. 49, p. 14-24, mayo 2013.

PAsA - Programa de Aprendizaje en Sexualidad y Afectividad. Sexualidades en la escuela. Santiago: PASA; Departamento de Psicología de la Universidad de Chile, 2012. Disponible en: <www.pasa.cl>. Acceso en: 10 sept. 2014.

Pontificia Universidad Católica de Chile. Teen Star. Santiago: PUC, 2012. Disponible en: <www.teenstar.cl>. Acceso en: 30 sept. 2014.

Rich, A. Desfazendo o "natural": a heterossexualidade compulsória e o continuum lesbiano. Revista Bagoas, Natal: Universidade Federal do Rio Grande do Norte, n. 5, p. 45-55, 2010.

Russell, S. T. Challenging homophobia in schools: policies and programs for safe scholl climates. Educar em Revista, Curitiba: UFPR, n. 39, p. 123-138, jan./abr. 2011.

Tin, L. G. Diccionario Akal de la Homofobia. Madrid: Ediciones Akal, 2012.

Unesco - Organización de las Naciones Unidas para la Educación, la Ciencia y la Cultura. Declaración Mundial sobre Educación para Todos y Marco de Acción para Satisfacer las Necessidades Básicas de Aprendizaje. Jomtien, Tailandia: 1990. Disponible en: $\langle$ http://www.unesco.org/education/pdf/JOMTIE_S.PDF $>$. Acceso en: 28 marzo 2018. 
Unesco - Organización de las Naciones Unidas para la Educación, la Ciencia y la Cultura. Foro Mundial sobre la Educación. Marco de Acción Dakar Educación para Todos: cumplir nuestros compromissos comunes. Dakar, Senegal: 2000. Disponible en: <http://unesdoc.unesco.org/images/0012/001211/121147s.pdf>. Acceso en: 28 marzo 2018.

. Respuestas del sector educación frente al bullying homofóbico. Cuadernillo 8. París: UNESCO, 2012.

Universidad Católica de la Santísima Concepción. Aprendiendo a Querer. Concepción: UCSC, 2012. Disponible en: <http://aprendiendoaquerer.cl>. Acceso en: 30 sept. 2014.

Universidad San Sebastián. Programa de Educación en Valores, Afectividad y Sexualidad —PAS. Santiago:USS, 2012. Disponible en: <www.uss.c1/pas/>. Acceso en: 30 sept. 2014. YAIKIN, B. Programas de Educación Sexual del MINEDUC incorporan plan que califica a la homosexualidad como "trastorno". El Dinamo, Chile, s.p., 6 jun. 2012.

\section{SOBRE EL AUTOR}

JuAn Rolando CoRnejo es doctor en ciencia política y doctor en estudios americanos por la Universidad de Santiago de Chile (Chile). Profesor de la Universidad Católica del Maule (Chile).

E-mail: jcornejo@ucm.cl

Recibido el 11 de marzo de 2016

Aprobado el 19 de julio de 2016 